\title{
Prediction Model of Triple play services for QoS Assessment in IP Based Networks
}

\author{
Jaroslav Frnda, Miroslav Voznak, Lukas Sevcik \\ VSB-Technical University of Ostrava, Ostrava, Czech Republic \\ Email: \{jaroslav.frnda@vsb.cz, miroslav.voznak, lukas.sevcik.st1\}@ vsb.cz \\ Peppino Fazio \\ University of Calabria, Cosenza, Italy \\ Email:pfazio@dimes.unical.it
}

\begin{abstract}
This paper brings a QoS (Quality of Service) assessment model which can estimate voice and video quality. Based on the proposed model, speech or video quality is computed with regard to congestion management QoS configuration in the network and its level of total utilization. The contribution of this paper lies in designing a new mathematical model capable of predicting the quality of multimedia services respecting network behavior and performance.
\end{abstract}

Index Terms - delay, E-Model, packet loss, QoS, SSIM, triple play.

\section{INTRODUCTION}

Network convergence that took place during the early 90 's of the last century, as well as appearance of NGN (Next Generation Network) concept, allowed the transfer of formerly separate services (voice, video and data) by one common network infrastructure. However, packet networks based on IP protocol had not been designed to transfer delay-sensitive traffic and difficulties appeared especially relating to voice because, without any supplementary mechanisms securing the quality of service, such a transfer was not capable of providing a high-quality interactive communication similar to standard TDM (Time Division Multiplex). Constant network monitoring, along with network performance intervening as needed, seems to be a method for securing at least minimal QoS level in the packet network. Therefore, the purpose of the model described in this paper is to provide a simple monitoring tool capable of predicting qualitative QoS parameters according to network status. The application aims to be an alternative to expensive monitoring tools, as well as a helpful tool for designing network infrastructure with regard to securing at least minimal QoS level.

\section{STATE OF THE ART}

Recently growing interest in voice and video transfer via packet networks based on IP protocol caused that analyses of these services and their behavior in such networks became more intensive. Logically, the highest emphasis is being put on the transfer of voice since this service is the most sensitive to an overall network status. References [1], [2] focus on degradation of voice service caused by delay and packet loss. These works use a simplified version of calculation model based on recommendation ITU-T G.107 (also known as E-model) [4] to evaluate the quality of speech, adjusting the model to be suitable especially for packet networks. At the same time, results are used to compare the application results to real practical experiments. Since the final delay and packet loss are factors depending on full network utilization and QoS policy applied to prioritized data flow processing by routers, it is necessary to consider this link as well. Related works [5-7] and [11] analyse in detail the impact of network utilization and usefulness of policies settings on a variable component of total delay. Congestion management features allow internet service provider to control congestion by determining the order in which packets are sent out an interface based on priorities assigned to those packets. Congestion management entails the creation of queues, assignment of packets to those queues based on the classification of the packet, and scheduling of the packets in a queue for transmission. Mechanisms of congestion management have been tested in full network traffic, and the results shown how these individual settings can influence overall quality. In [8] especially the impact of the buffer size for Jitter and packet loss in the network is being studied. Our paper compares different levels of network utilizations, thus extends the previous works and adds video quality measurements as a part of complex comparison.

Wireless networks have become a subject of immense interest in the recent years. Work [13] focused on the problem of quality of service over wireless interfaces and proposed the vertical QoS mapping concept. In the other work [19], they offer a different view on speech quality in wireless environment. The key issue is to find a suitable method to dynamically obtain a subjective measure of user QoE (Quality of Experience) in an on-going service from the subjective measures. Model described there provides a quick way to estimate QoE from objective QoS parameters. The analysis of video quality focuses on resistance of video codecs towards packet loss in the network that cause the artifacts in video [9], [10], [17]. 
The measurements in these works show dependence of resolution, bitrate and codec type on decrease of video quality through increasing packet loss rate in network the higher the bitrate and resolution are, the better their resistance towards unwanted effects is. Paper [16] presents the tool based on the adaptation of the transmission rate and acts on the basis of feedback information about the overall network status estimated in terms of maximum allowable network throughput and link quality.

But, what is still missing, though, is the application of many experiments results to real use. Therefore, this paper attempts to bring a tool which, according to the application of mathematical models based on actual results, would be capable of providing with reliable information on Triple play services quality.

\section{Methodology}

This paper follows directly our previous experiments studying the impact of full network utilization and performance of data prioritization on the final quality of service [5]. In [5], we introduced and described tools enabling analysis of quality of triple-play services. In this work, we focused on improvements and addition of a model calculation. Next additional measurements were carried out which brought more accurate mathematical model. For further upgrade of the model, it was essential to analyse the link between different level of network utilization and Triple play services quality (mainly the quality of voice and video).

In order to generate VoIP calls ( 5 for each test and every test has been performed 10 times), the tool IxChariot from Ixia company was used. IxChariot provides the ability to assess the performance characteristics of any application running on wired and wireless networks especially in our case for the purpose of VoIP QoS performing tests.

As a first step of video quality testing, we created a stream in the VLC Player. As for the video content, streaming process RTP/UDP/IP method with MPEG2(TS) and H.264(MP4) was used. We broadcasted the video stream via testing network topology and captured and saved the stream to disk for comparison with original video file.

Linux tool iperf was used for utilization of the network to specific levels as is depicted in Fig.1. Iperf allows to measure maximum TCP and UDP bandwidth, calculates delay, jitter and datagram loss.

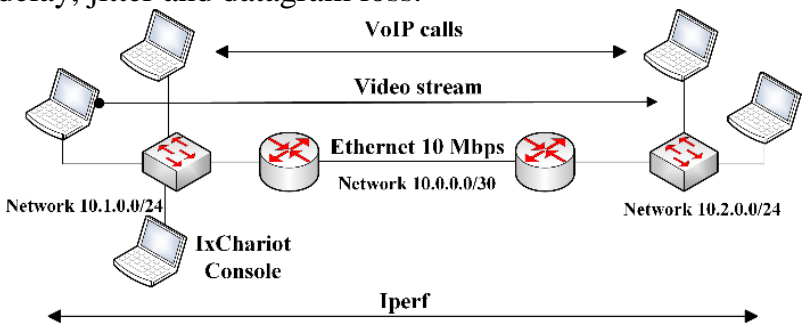

Figure 1. Measurement scheme
In order to transfer multimedia time-sensitive services without additional delay, only UDP protocol is used. The unreliable UDP protocol makes no effort to set up a connection, it does not check to see if the data was received and usually does not make any provisions for recovering from errors or lost data.

Factors affecting the multimedia transmission are:

- Latency - This is the time that elapses between sending a message from the source and adoption of the destination node.

- Packet order (Jitter) - Variability in the packet delivery time to the target node causes incorrect order.

- Packet loss - Occurs when one or more packets of data traveling across a computer network fail to reach their destination. It is most often expressed as a percentage.

- Bandwidth - This expresses the capacity of the transmission channel.

- Delay - This is caused by overcrowding the packet queue on the outgoing interface [5].

MSU VQMT tool served to compare a streamed video with the original one so that the impact of network utilization could be analysed. Implementation of the computational model was carried out in programming language $\mathrm{C \#}$.

\section{A. Measured Parameters}

In E-model, defined by Recommendation G.107 by ITU-T [4], was used as an objective evaluation method for voice service. This model is based on the calculation of R-factor, as follows:

$$
R=R_{O}-I_{S}-I_{D}-I_{E-E F F}+A .
$$

in which $R_{O}$ is a basic signal-to-noise ratio, $I_{S}$ is a sum of all degradation factors appearing simultaneously with the voice transfer, then $I_{D}$ represents degradation caused by the voice transfer delay, $I_{E-E F F}$ is a factor representing the impairment of quality caused by packet loss and finally, the value $A$ represents an advantage factor, that depends on the type of communication system.

TABLE I

EXAMPLES FOR THE ADVANTAGE FACTOR A [4]

\begin{tabular}{|l|c|}
\hline \multicolumn{1}{|c|}{ Communication system example } & Maximum value of A \\
\hline Conventional (wire bound) & 0 \\
\hline Mobility by cellular networks in a building & 5 \\
\hline $\begin{array}{l}\text { Mobility in a geographical area or moving in } \\
\text { a vehicle }\end{array}$ & 10 \\
\hline $\begin{array}{l}\text { Access to hard-to-reach locations, e.g. via } \\
\text { multi-hop satellite connections }\end{array}$ & 20 \\
\hline
\end{tabular}

Previously mentioned recommendation also includes a set of recommended values that enable to simplify the calculation, so that it corresponds to packet networks. If 
all parameters are set to the default values, the calculation results in a very high quality with a rating factor of $\mathrm{R}=$ 93.35 [4]. In order to meet user's expectations, the value of 70 or more is needed [1]. From the referred network affecting parameters, regarding to the QoS in packet networks, we focused especially on network delay, Jitter and packet loss. These parameters are included in two factors used to calculate the R-factor, in $I_{D}$ (2) and in above mentioned $I_{E-E F F}[4]$.

$$
I_{D}=I_{D T E}+I_{D D}
$$

The parameter $I_{D T E}$ represents the factor of impairment caused by echo (Echo-cancellation has been solved in ITU-T G.168 recommendation), and $I_{D D}$ represents the factor of impairment caused by too long transfer delay. Simplified calculation of the R-factor uses the following final formula:

$$
\mathrm{R}=93.35-\mathrm{I}_{\mathrm{D}}-\mathrm{I}_{\mathrm{E}-\mathrm{EFF}} \cdot
$$

Except from the R-factor values, a rating scheme of 1 5 (5 is the best), called MOS (Mean Opinion Score), can be used as an evaluation scale. Conversion of the R-factor values to MOS scale values is described in previously mentioned recommendation.

In the work, we deal with objective methods for evaluation of the quality of video sequences. Subjective assessment methods are very demanding regarding the time and people, and comparison of each other is not a simple process. The objective methods are commonly preferred and offer the results that can be used for immediate comparison and verification. Each used objective method has a different procedures and different metric evaluation system. We used two objective methods - PSNR and SSIM. Objective evaluation metric involves the use of the metric's computational methods, which form a "score" of the quality of the investigated video, see Fig.2. These methods measure the physical characteristics of the video signal, such as the amplitude, timing and signal-to-noise ratio.

PSNR is defined via the mean squared error (MSE) which represents squared deviation between a tested and an original sample and the maximum possible pixel value of the image as follows: [10]:

$$
P S N R=10 \log _{10}\left(\frac{M A X_{I}^{2}}{M S E}\right),[d B] .
$$

where MAX is the maximum value that the pixel can take (e.g. 255 for 8-bit image), and MSE is the difference between two grey-level images or video sequences. Technically, MSE reflects the diversity of the image while PSNR expresses its identity. The strongest PSNR method is an easy and fast calculation, which is the reason why it is still used very often in scientific papers although the correlation with the human perception is worse than SSIM [6], [8].

The SSIM method considers perception of image by the human eye. It measures the luminance, contrast, and structural similarity and combines them into one result value -determine the video quality - interval $[0,1] ; 0$ the worst and 1 the best quality. Final SSIM value is a combination of three parameters, with original signal $\mathrm{x}$ and encoded signal y being defined as follows [9], [14]:

$$
\operatorname{SSIM}(x, y)=[l(x, y)]^{\alpha}[c(x, y)]^{\beta}[s(x, y)]^{\gamma}
$$

- Element $\mathbf{l}(\mathbf{x}, \mathbf{y})$ compares the brightness of the signal

- Element $\mathbf{c}(\mathbf{x}, \mathbf{y})$ compares the contrast of the signal

- Element $\mathbf{s}(\mathbf{x}, \mathbf{y})$ measures the structure of correlation

- $\alpha>0, \beta>0, \gamma>0$ measures the weight of individual elements

Four levels of utilization were used to analyse the dependence of network utilization on the quality of voice and video $-25 \%, 50 \%, 75 \%$ and $100 \%$. The voice service was represented by two codecs with the highest assessment according to PESQ ranking - G.711 A-law and G.729. The video service was represented by two currently the most used video codecs for digital broadcasting - MPEG-2 and MPEG-4(h.264). Routers were not set to any specific data processing policy (Best

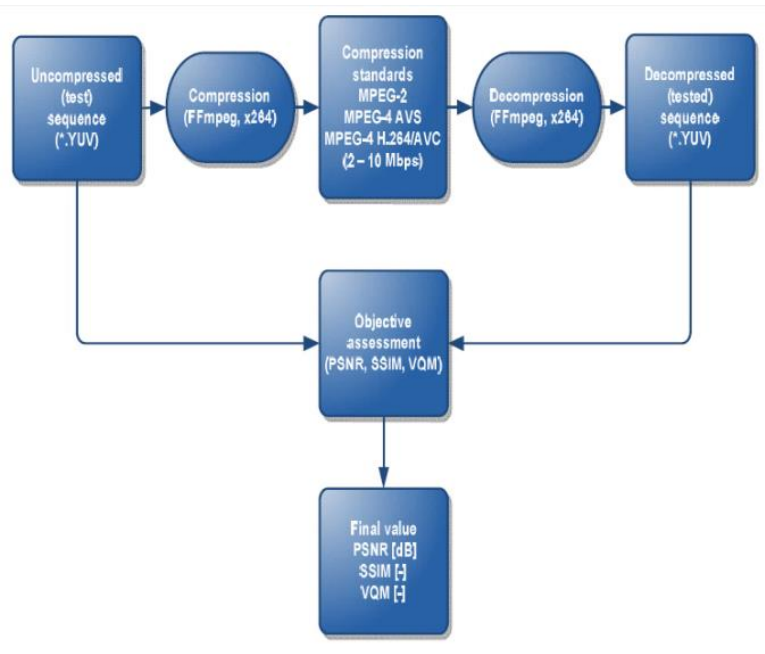

Figure 2. Scheme for objective assessment of video

Effort). Ethernet bandwidth consumption of voice codec G.711 is approximately 3 times bigger than G.729 (90.4 vs. $34.4 \mathrm{kbps})$. Codec G.729 also contains an implementation of PLC algorithm, hence offers better resistance during the higher level of packet loss in the network [6].

For the purposes of mathematical formulation of the $\mathrm{R}$-factor values drop with regard to the level of network utilization, it is essential to analyse the ratio of constituent factors $I_{E-E F F}$ and $\mathrm{I}_{\mathrm{D}}$ (packet loss and total mouth to ear-delay) at such occurrence.

The decrease in QoS, altogether with the analyzed impact of packet loss on the final quality of the video, was processed into following regressive equations in which $\mathrm{X}$ stands for percentage packet loss ratio in network. Verification of these mentioned formulas are published in our paper [5]. These formulas were extended by the analyses of impact of video type sequences. We made measurements for one static and two dynamic videos of 25 seconds. All the movies were measured at a 
resolution of $720 \times 576$ (PAL), $1280 \times 720$ (HD) and 1920x1080 (Full HD). Static video was represented by TV news (slow motion), the first dynamic video by a space shuttle launch and the third video with the highest bitrate $(60 \mathrm{Mbps})$ by an open source animated movie called Big Buck Bunny.

\section{B. Features of Implemented SW Tool Designed According to Simulation Model}

Graphical environment of the application appears when you

start the computational model (Fig. 3). It is divided into four logical parts: the main menu, the orange frame, gray frame, light blue frame. The main menu is used to set the language or select QoS policies; the best effort method is chosen by default. Orange frames are used for the selection of voice or video codecs and network utilization. Using gray frames can specify the network parameters and light blue frames enables to display results of the simulation. Since the application focuses on the whole scale of Triple play services, there is one service left - data. We have dealt with this service as well in previously mentioned paper [5], which detected the ratio between network capacity and actually reached speed of ftp service with regard to utilization and implemented QoS policy. Overall application settings:

- Calculation of MOS and the R-factor, based on

- prediction of total delay, Jitter buffer, selected packet

- loss and network utilization

- Calculation of SSIM and PSNR for video with regard

- to selected packet loss in the network

- Bandwidth for data service within Triple-play

- Following parameters are possible to set for the purposes of

- application settings the specification:

- Bandwidth uplink and downlink at end-user

- Number of routers, their configured policies and

- utilization of individual sections

- Distance between communicating points

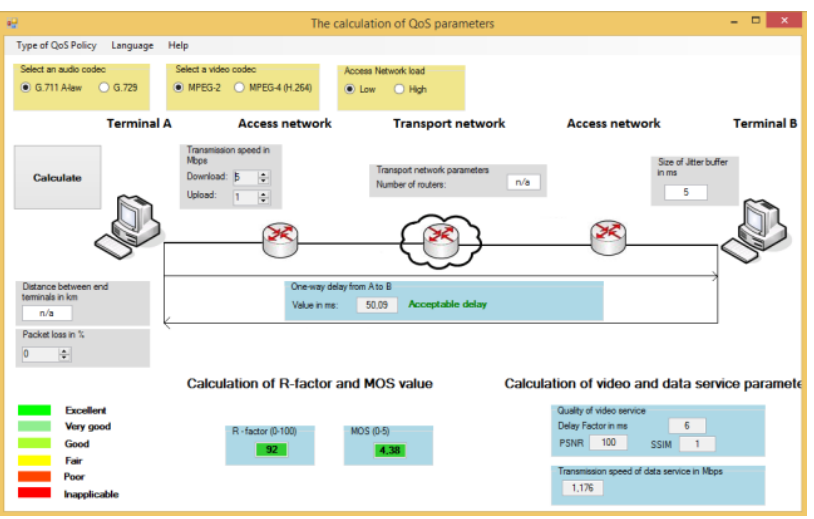

Figure 3. Graphical design of the application
The application offers as next optional parameters the total delay, De-Jitter buffer size and packet loss values (if not filled then default values are used: $0,60,0)$.

\section{MEASUREMENTS AND RESULTS}

The first step of our research was to analyse the impact of network utilization on quality of voice service. Following Table II gives information about the measurement results. As it can be seen, the network utilization has a significant impact only when the network link capacity reached its top.

TABLE II

DEPENDENCE OF NETWORK UTILIZATION AND QOS FOR THE VOICE

\begin{tabular}{|l|c|c|c|c|}
\hline \multirow{2}{*}{ Network utilization } & \multicolumn{2}{|c|}{ MOS } & \multicolumn{2}{c|}{ R-factor } \\
\cline { 2 - 5 } & G.711 & G.729 & G.711 & G.729 \\
\hline Only 5 calls & 4.37 & 4.03 & 91.45 & 81.31 \\
\hline $25 \%$ & 4.33 & 4.03 & 90.22 & 80.15 \\
\hline $50 \%$ & 4.28 & 4.03 & 88.92 & 80.1 \\
\hline $75 \%$ & 3.44 & 3.99 & 68.49 & 76.96 \\
\hline $100 \%$ & 2.92 & 3.89 & 56.81 & 76.08 \\
\hline
\end{tabular}

Another group of measurements was carried out to express the $I_{E-E F F}$ factor, using Linux tool netem. Netem provides Network Emulation functionality for testing protocols by emulating the properties of wide area networks. The current version emulates variable delay, loss, duplication and reordering.

We set the packet loss to $1 \%$ on the interface using Netem and then repeated the whole measurement. Then we repeated this step for packet loss in increments of $1 \%$, $2 \%, \ldots, 10 \%$. The results of measurements showed following regressive equations:

For codec G.711 PCM

$$
Y=\sqrt{(a+(b * X))}, \quad R^{2}=99.37 \%
$$

$\mathrm{Y}=\mathrm{I}_{\mathrm{E}-\mathrm{EFF}} \quad \mathrm{X}=$ packet loss $(\%)$

$\mathrm{a}=-207.44 \quad \mathrm{~b}=536.251$

For codec G.729 CS-ACELP

$$
Y=\sqrt{(a+(b * X))}, \quad R^{2}=99.34 \%
$$

$\mathrm{Y}=\mathrm{I}_{\mathrm{E}-\mathrm{EFF}} \quad \mathrm{X}=$ packet loss $(\%)$

$\mathrm{a}=91.1429 \quad \mathrm{~b}=157.535$

Diagrams depicted in Fig. 4 and Fig. 5 describe functions of designed models, along with correspondence comparison to results from other papers. According to the charts, better results for G.729 can be seen in the case of bigger data loss. 


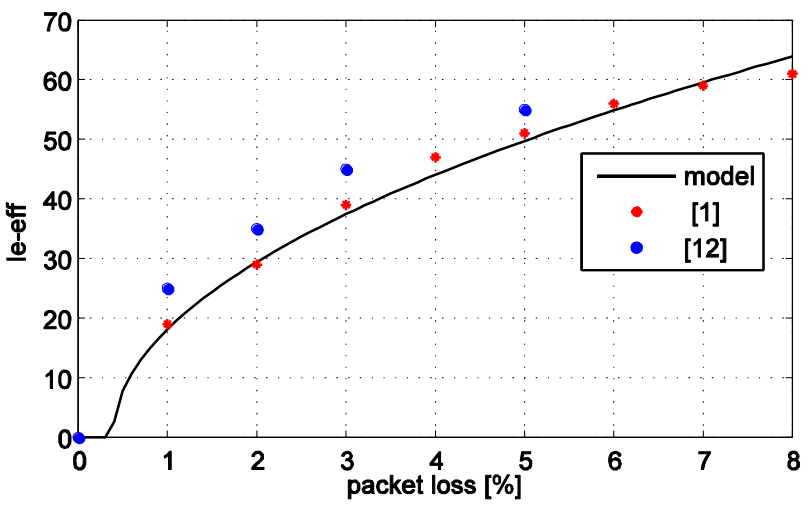

Figure 4. $\mathrm{I}_{\mathrm{E}-\mathrm{EFF}}$ factor as a function for G.711

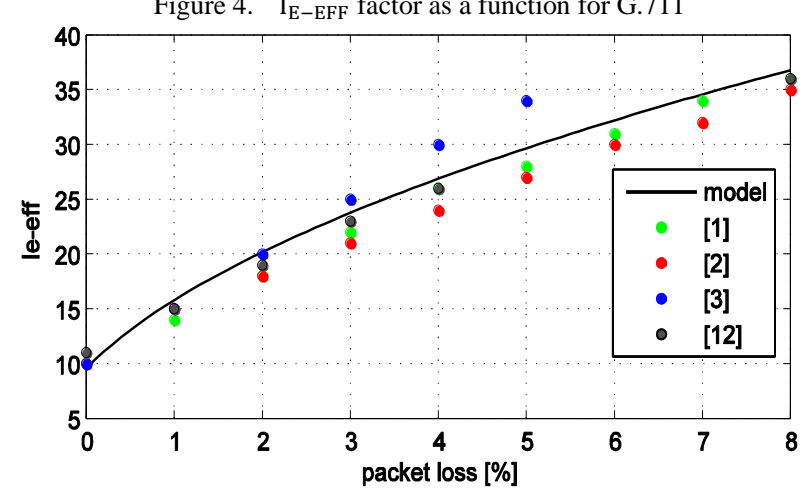

Figure 5. $\mathrm{I}_{\mathrm{E}-\mathrm{EFF}}$ factor as a function for G.729

The second factor $I_{D}$, is being expressed by regressive equation used in our previous work and specified at the beginning of the interval, as follows [5]:

For $\mathrm{I}_{\mathrm{D}}$ at the interval of $0-180 \mathrm{~ms}$

$$
Y=\left(a+\left(b * X^{2}\right)\right)^{2} .
$$

$$
\begin{array}{ll}
\mathrm{Y}=\mathrm{I}_{\mathrm{D}} & \mathrm{X}=\text { total delay }(\mathrm{ms}) \\
\mathrm{a}=-0.606899 & \mathrm{~b}=0.0000602265
\end{array}
$$

For $\mathrm{I}_{\mathrm{D}}$ at the interval from $180 \mathrm{~ms}$

$$
Y=\left(a+\frac{b}{x}\right)^{2}, \quad R^{2}=99.83 \%
$$

$$
\begin{array}{ll}
\mathrm{Y}=\mathrm{I}_{\mathrm{D}} & \mathrm{X}=\text { total delay }(\mathrm{ms}) \\
\mathrm{a}=7.87441 & \mathrm{~b}=-1192.81
\end{array}
$$

If both of these factors are specified, it is possible to check the equation (3) for realized measures. Table III describes the results of variable delay for two routers through which the network was utilized, along with $I_{D}$ and $\mathrm{I}_{\mathrm{E}-\mathrm{EFF}}$ factors. The relative standard deviation (\%RSD) is a statistical measure of the precision for a series of repetitive measurements. The RSD is calculated from the standard deviation and is commonly expressed as a percentage $(\%)$. Due to the very low calculated values of RSD, it can be pronounced that mentioned regressive equations implemented for the application are truly accurate. An interesting fact is that the delay caused by two routers is quite big $(94-38=56 \mathrm{~ms})$ already at network utilization of $50 \%$.

TABLE III

CALCULATION OF THE R-FACTOR BY IMPLEMENTING EQUATIONS AND VALUES OBTAINED BY MEASUREMENTS

\begin{tabular}{|l|c|c|c|c|c|c|}
\hline $\begin{array}{l}\text { Network } \\
\text { utilization }\end{array}$ & Codec & $\begin{array}{c}\text { Variable } \\
\text { delay }\end{array}$ & $\begin{array}{c}\text { End-to- } \\
\text { End } \\
\text { delay }\end{array}$ & $\mathrm{I}_{\mathrm{D}}+\mathrm{I}_{\mathrm{E}-\mathrm{EFF}}$ & $\begin{array}{c}\mathrm{R}- \\
\text { factor }\end{array}$ & $\%$ RSD \\
\hline \multirow{2}{*}{$50 \%$} & G.711 & $94(38)$ & 154 & 4.19 & 89.16 & 0.27 \\
\cline { 2 - 7 } & G.729 & $98(41)$ & 173 & 12.14 & 81.21 & 1.39 \\
\hline \multirow{2}{*}{$75 \%$} & G.711 & $120(49)$ & 181 & 25.41 & 67.94 & 0.8 \\
\cline { 2 - 7 } & G.729 & $121(51)$ & 196 & 15.74 & 77.61 & 0.84 \\
\hline \multirow{2}{*}{$100 \%$} & G.711 & $145(56)$ & 206 & 37.49 & 55.86 & 1.67 \\
\cline { 2 - 7 } & G.729 & $146(55)$ & 221 & 19.17 & 74.18 & 2.5 \\
\hline
\end{tabular}

NOTE: NUMBER IN BRACKETS DETERMINES THE SIZE OF JITTER WITHIN VARIABLE DELAY; DELAY VALUES ARE EXPRESSED IN MILLISECONDS

The conclusion from [6] is confirmed, since it emphasizes the importance of network performance type. During our measurements, we were equipped not only with voice calls in network performance, but also with a streamed video and several UDP flows generated by iperf. Such a huge data extent resulted in a decrease of network efficiency already at its half-sized utilization. The delay values on routers acquired by measurements, as well as Jitter value, will be applied for the calculation of total delay with regard to number of used routers and network utilization at individual sections expressed by percentage (0\% 50\% 75\% 100\%).

For the calculation of buffer size to eliminate Jitter delay, minimally 1.5 times larger size of the buffer, compared with measured average delay, is required [15]; the application is based on this recommendation. In order to use an adequate delay value on routers with regard to network section utilization, a simple equation with measured values can be implemented for the application (9):

$$
R_{D}=44.5963+45.3578^{*}(X)^{2}
$$

In which $R_{D}$ stands for delay on the two routers and $\mathrm{X}$ stands for utilization of the section $(0.5 ; 0.75 ; 1)$. When implementing QoS policy other than Best Effort, results of measurements are processed [5].

The last delay component that needs to be focused on is Jitter, or more precisely the buffer size on receiving side. In case the buffer is set at a low level, its fast filling is usually followed by packet drop. On the other hand, setting the buffer on too high level causes a disproportionate increase of one-way delay. The application based on previously mentioned recommendation and results acquired by measurements calculates the appropriate buffer size. It also offers an option to set any buffer size. However, it is necessary to consider this factor when making an overall evaluation of final quality of service. For the purposes of such an evaluation, following Table IV was compiled, using the results from [8]. 
TABLE IV

IMPACT OF BUFFER SIZE

\begin{tabular}{|l|c|c|}
\hline $\begin{array}{l}\text { Jitter } \\
{[\mathrm{ms}]}\end{array}$ & $\begin{array}{c}\text { Buffer } \\
\text { size [ms] }\end{array}$ & $\begin{array}{c}\text { Average buffer loss } \\
{[\%]}\end{array}$ \\
\hline 20 & 40 & 0.1 \\
\hline 20 & 60 & 0.03 \\
\hline 40 & 40 & 5.98 \\
\hline 40 & 60 & 1.45 \\
\hline 80 & 40 & 14.75 \\
\hline 80 & 60 & 7.9 \\
\hline
\end{tabular}

The Table IV shows that if the buffer is more than double-sized, there is a minimal chance of packet loss increase. On the contrary, if the buffer size is approximately the same or smaller than average Jitter, it results in significant packet loss increase. The ratio between Jitter and buffer size may be expressed as follows:

$$
P_{l b}=-4.65399+9.71812 * X .
$$

The result $\mathrm{P}_{\mathrm{lb}}$ represents the increase of packet loss in the network (\%) and $\mathrm{X}$ represents the ratio between Jitter and buffer on the scale $0.5-2$.

The results of quality of video measurements stress an enormous impact of packet loss in the network. Since streamed broadcasting via the Internet known as IPTV is a one-way service, the delay does not play such a significant role in this case. Newer codec MPEG-4 uses stronger compression than its predecessor. All movies were measured in a resolution of $720 \times 576$ (PAL), 1280 x 720 (HD) and 1920 x 1080 (Full HD). Static video was represented by TV news (slow motion), the first dynamic video by a NASA space shuttle launch, and the third video with the highest bitrate (70 Mbps in full HD) was an open source animation movie for testing purposes called Big Buck Bunny. To evaluate the quality, we used the methods PSNR and SSIM. We evaluated these methods using MSU VQM Tools. As a first step, we created a stream in the VLC Player. As for the video content, streaming process RTP/UDP/IP method with MPEG2 (TS) and H.264 (MP4) was used.

We captured the broadcast stream on the local computer interface using VLC Player. This video was saved and tagged as the original video.

TABLE V

DEPENDANCE OF NETWORK UTILIZATION AND QOS FOR VIDEO SERVICE

\begin{tabular}{|l|r|r|r|r|}
\hline \multirow{2}{*}{$\begin{array}{l}\text { Network } \\
\text { utilization }\end{array}$} & \multicolumn{2}{|c|}{ MPEG-2 } & \multicolumn{2}{c|}{ MPEG-4 (h.264) } \\
\cline { 2 - 5 } & PSNR [dB] & \multicolumn{1}{c|}{ SSIM } & PSNR [dB] & SSIM \\
\hline Only 1 stream & 51.835 & 0.989 & 51.235 & 0.989 \\
\hline $25 \%$ & 49.124 & 0.985 & 46.511 & 0.965 \\
\hline $50 \%$ & 47.16 & 0.976 & 32.001 & 0.949 \\
\hline $75 \%$ & 40.72 & 0.961 & 24.531 & 0.919 \\
\hline $100 \%$ & 27.234 & 0.929 & 17.036 & 0.763 \\
\hline
\end{tabular}

While streaming the video that demands much higher capacity than voice, more significant packet loss was detected. The values of packet loss for both, MPEG-2 and MPEG-4, expressed in percentage, can be seen in Table VI.
TABLE VI

PACKet Loss VALUes DURING THE TeSTING OF VIDEO SERVICES

\begin{tabular}{|l|c|c|c|c|c|}
\hline Codec & $\begin{array}{c}\text { Only 1 } \\
\text { stream }\end{array}$ & $25 \%$ & $50 \%$ & $75 \%$ & 100 \\
\hline MPEG-2 & 0 & 0.06 & 0.1 & 0.31 & 4.78 \\
\hline MPEG-4 & 0 & 0.05 & 0.16 & 0.36 & 9.45 \\
\hline
\end{tabular}

The decrease in QoS, altogether with the analysed impact of packet loss on the final quality of the video, was processed into following regressive equations in which $\mathrm{X}$ stands for percentage packet loss ratio in network. Regressive equations that were published in [18] are extended, and better specified for particular video sequence type and resolution.

- Slow-motion video.

\section{MPEG-2}

$\operatorname{SSIM}=\alpha\left(a+b *\left(X^{2}\right)\right)+\beta(a+b * \sqrt{X})+\gamma\left(a+b *\left(X^{2}\right)\right)$

\section{MPEG-4}

$\left.\operatorname{SSIM}=\alpha\left(\sqrt{a+\frac{b}{X}}\right)\right)+\beta\left(\frac{1}{a+b * X}\right)+\gamma(\exp (a+b * X))$.

All the necessary coefficients are presented in Table VII.

TABLE VII

COEFFICIENTS FOR STATIC VIDEO

\begin{tabular}{|c|c|c|c|c|c|c|}
\hline \multirow{2}{*}{$\begin{array}{c}\text { Co } \\
\text { ef. }\end{array}$} & \multicolumn{3}{|c|}{ MPEG-2 } & \multicolumn{3}{c|}{ MPEG-4 (H.264) } \\
\cline { 2 - 7 } & $720 \times 576$ & $\begin{array}{l}1280 \\
\mathrm{x} 720\end{array}$ & $\begin{array}{l}1920 \\
\mathrm{x} 1080\end{array}$ & $720 \times 576$ & $\begin{array}{l}1280 \\
\mathrm{x} 720\end{array}$ & $\begin{array}{l}1920 \\
\mathrm{x} 1080\end{array}$ \\
\hline $\mathrm{a}$ & 0.89957 & 1.08748 & 0.9216 & 0.146704 & 1.1027 & 0.08596 \\
\hline $\mathrm{b}$ & -0.004924 & -0.143973 & -0.00389 & 0.528499 & 0.12312 & - \\
\hline$\alpha$ & 1 & 0 & 0 & 1 & 0 & 0 \\
\hline$\beta$ & 0 & 1 & 0 & 0 & 1 & 0 \\
\hline$\gamma$ & 0 & 0 & 1 & 0 & 0 & 1 \\
\hline
\end{tabular}

Because measurements were performed on two dynamic videos, the following regressive equations represent a prediction model for both of them.

- Dynamic video with ordinary and high bitrate. MPEG-2

$$
\operatorname{SSIM}=\alpha\left(\frac{1}{a+b * X}\right)+\beta(a+b * \ln (X)) .
$$

MPEG-4

$$
\operatorname{SSIM}=\alpha(a+b * \ln (X))+\beta\left(\frac{1}{a+b * X}\right) .
$$

Tables VIII and IX contain the coefficients for these two equations. All regressive models described here gained an R-square factor $\left(R^{2}\right)$ higher than $90 \%$, which represents a high level of veracity. 
TABLE VIII

COEFFICIENTS FOR MPEG-2 DYNAMIC VIDEOS

\begin{tabular}{|c|c|c|c|c|c|c|}
\hline \multirow{2}{*}{$\begin{array}{l}\text { Co } \\
\text { ef. }\end{array}$} & \multicolumn{3}{|c|}{ Lower bitrate dynamic video } & \multicolumn{3}{|c|}{ High bitrate dynamic video } \\
\hline & $720 \times 576$ & $\begin{array}{l}1280 \\
\times 720\end{array}$ & $\begin{array}{l}192 \\
\mathrm{x} 1080\end{array}$ & $720 \times 576$ & $\begin{array}{l}1280 \\
\times 720\end{array}$ & $\begin{array}{l}1920 \\
\mathrm{x} 1080\end{array}$ \\
\hline $\mathrm{a}$ & 0.858125 & 0.875076 & 0.927705 & 0.9538 & 0.924954 & $\begin{array}{c}0.81 \\
9482\end{array}$ \\
\hline $\mathrm{b}$ & -0.076882 & -0.080159 & -0.00389 & 0.094724 & -0.179784 & $\begin{array}{r}- \\
0.1216\end{array}$ \\
\hline$\alpha$ & 0 & 0 & 0 & 1 & 0 & 0 \\
\hline$\beta$ & 1 & 1 & 1 & 0 & 1 & 1 \\
\hline
\end{tabular}

TABLE IX

COEFFICIENTS FOR MPEG-4(H.264) DYNAMIC VIDEOS

\begin{tabular}{|c|c|c|c|c|c|c|}
\hline \multirow{2}{*}{$\begin{array}{c}\text { Co } \\
\text { ef. }\end{array}$} & \multicolumn{3}{|c|}{ Lower bitrate dynamic video } & \multicolumn{3}{c|}{ High bitrate dynamic video } \\
\cline { 2 - 7 } & $720 \times 576$ & $\begin{array}{l}1280 \\
\mathrm{x} 720\end{array}$ & $\begin{array}{l}1920 \\
\mathrm{x} 1080\end{array}$ & $720 \times 576$ & $\begin{array}{l}1280 \\
\mathrm{x} 720\end{array}$ & $\begin{array}{l}1920 \\
\mathrm{x} 1080\end{array}$ \\
\hline $\mathrm{a}$ & 0.783421 & 1.12248 & 0.800991 & 0.678364 & 1.8151 & 00.5818 \\
\hline $\mathrm{b}$ & -0.073105 & 0.086301 & -0.092020 & -0.169198 & 0.122547 & -0.0833 \\
\hline$\alpha$ & 1 & 0 & 0 & 0 & 0 & 0 \\
\hline$\beta$ & 0 & 1 & 1 & 1 & 1 & 1 \\
\hline
\end{tabular}

\section{CONCLUSION}

The aim of this work is to bring a comprehensive look at Triple-play services and their demands on the network infrastructure. The resulting new simulating model is based on real measurements of experiments and is able to predict objective QoS parameters of Triple play services in IP network. With the developed application based on the proposed model, you can easily and quickly see the impact of the use of QoS policies and network parameters for objective evaluation techniques of Triple-play services. The accuracy of mathematical models was verified through a method of comparing the calculation with results of actual experiments. Measured results then served as predefined scenarios concerning packet loss in network, delay at particular policy implementation and selected network utilization. The use of proposed models provides the application with an ability to react immediately to any changes in default settings and adapt to a specific status as much as possible. Further improvement of the application is expected. The next step should be an analysis of the impact of security and coding mechanisms implemented on QoS parameters. Security is a highly discussed topic nowadays, and protocols such as IPsec, SSL/TLS, SRTP or ZRTP are becoming more and more frequently used to secure the content of voice or video. Therefore, the proposed analytical computational model should take into account various security measures in network as well.

\section{ACKNOWLEDGMENT}

This research was funded by the grant SGS reg. no. SP2015/82 conducted at VSB-Technical University of Ostrava, Czech Republic and was partially supported by the European Regional Development Fund in the IT4Innovations Centre of Excellence project (CZ.1.05/1.1.00/02.0070) and by the Development of human resources in research and the development of latest soft computing methods and their application in practice project (CZ.1.07/2.3.00/20. 0072) funded by the Operational Programme Education for Competitiveness.

\section{REFERENCES}

[1] M. Voznak, "E-model modification for the case of cascade codecs arrangement," International Journal of Mathematical Models and Methods in Applied Sciences, vol. 5, no. 8, 2011, pp. 1439-1447.

[2] R.G. Cole and J. H. Rosenbluth, "Voice over IP performance monitoring," ACM SIGCOMM Computer Communication, 2001, vol. 31, no. 2, pp. 9-24.

[3] M. Marchese, "Quality of Service over Heterogeneous Networks", Wiley \& Sons Ltd, Chichester, England, ISBN 978-0-470-01752-4 (HB), 2007.

[4] ITU-T G.107, The E-model, a computational model for use in transmission planning, ITU-T Recommendation G.107, ITU-T Geneva, Switzerland: May 2010.

[5] J. Frnda, M. Voznak, J. Rozhon, M. Mehic, "Prediction Model of QoS for Triple Play Services," 21st Telecommunications Forum TELFOR 2013, pp. 733-736.

[6] Mansour J. Karam, Fouad A. Tobagi, "Analysis of delay and delay jitter of voice traffic in the Internet," Computer Networks 40, pp. 711-726.

[7] F. De Rango, P. Fazio, F. Conte, F. Scarcello, "A New Distributed Application and Network Layer Protocol for VoIP in Mobile Ad Hoc Networks," in IEEE Transactions on Mobile Computing, 2014, pp. 2185-2198.

[8] A. Kovac, M. Halas, "E-model mos estimate precision improvement and modelling of jitter effects," Advances in Electrical and Electronic Engineering, vol. 10, no. 4, 2012, pp. 276-281.

[9] Y. Changhoon and A. C. Bovik, "Evalution of temporal variation of video quality in packet loss networks." Signal Processing: Image Communication, 2011, pp 34-38.

[10] M. Uhrina, J. Hlubik, M. Vaculik, "Impact of Compression on the Video Quality." In Journal Advances in Electrical and Electronic Engineering, vol. 10, no. 4, 2012, pp. 251258.

[11] Braun, T., Diaz, M., Gabeiras, J.E., Staub, "The End-toEnd Quality of Service Over Heterogeneous Networks", Springer, ISBN 978-3-540-79119-5, 2008.

[12] F. De Rango, F. Guerriero, P. Fazio, "Link-stability and energy aware routing protocol in distributed wireless networks," in IEEE Transactions on Parallel and Distributed Systems, vol. 23, no. 4, 2012, pp. 713-726.

[13] M. Marchese, M. Mongelli, "Vertical QoS Mapping over Wireless Interfaces", IEEE Wireless Communications Magazine, vol. 16, no. 2, 2009, pp. 37-43.

[14] Z. Wang, E.P. Simoncelli and A.C. Bovik, "Multi-scale structural similarity for image quality assessment," In Proc. Signals, Systems and Computers, vol. 2, 2004, pp.13981402.

[15] ITU-T G.113, Transmission impairments due to speech processing, ITU-T Recommendation G.113, ITU-T Geneva, Switzerland. 2001.

[16] I. Bisio, F. Lavagetto, G. Luzzati, M. Marchese, "Smartphones Apps Implementing a Heuristic Joint Coding for Video Transmissions over Mobile Networks", Mobile Networks and Applications, 2014, vol. 19, no. 4, pp. 552-562.

[17] M. Uhrina, J. Hlubik, M. Vaculik, "Correlation Between Objective and Subjective Methods Used for Video Quality Evaluation," In Journal Advances in Electrical and Electronic Engineering, vol 11, no. 2, 2013, pp. 135-146. 
[18] L. Sevcik, M. Voznak, J. Frnda, “QoE Prediction Model for Multimedia Services in IP Network Applying Queuing Policy," International Symposium on Performance Evaluation of Computer and Telecommunication Systems, 2014, pp. 593-598.

[19] J.A. Hassan, M. Hassan, S. K. Das, A. Ramer, "Managing Quality of Experience for Wireless VOIP Using Noncooperative Games", Selected Areas in Communications, IEEE Journal on, vol. 30, no. 7, 2012, pp. 1193-1204.

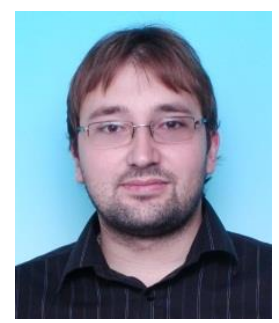

Jaroslav Frnda (1989) received his M.Sc. from the Faculty of Electrical Engineering and Computer Science, VSB-Technical University of Ostrava, in 2013. He is continuing his Ph.D. study at the Dept. of Telecommunications in the same faculty. His research interests include Next Generation Networks and objective speech and video quality

assessment methods.

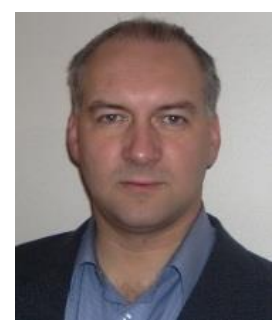

Miroslav Voznak (1971) is an associate professor and department chair, at the Dept. of Telecommunications, VSBTechnical University of Ostrava. He received his $\mathrm{Ph} . \mathrm{D}$. in telecommunications in 2002 from Faculty of electrical Engineering and Computer Science, VSB-TU Ostrava, and his dissertation thesis was entitled "Voice traffic optimization with regard to speech quality in networks with VoIP technology". Topics of his research interests are Next Generation Networks, IP telephony, speech quality and network security.

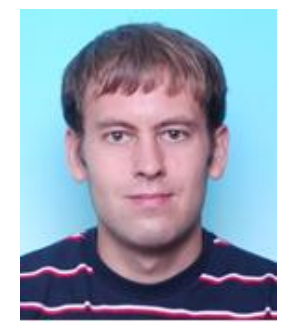

Lukas Sevcik (1989) received his M.Sc in Informatics from the Faculty of Management Science and Informatics, University of Zilina, in 2013. Now, he is continuing his Ph.D. study at the Dept. of Telecommunications, VSB-Technical University of Ostrava. His research interests include Quality of Triple play services and IP networks.

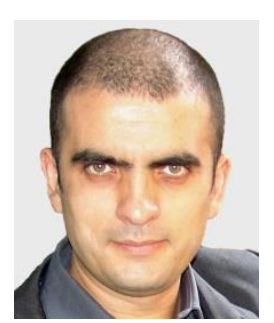

Peppino Fazio received the master's degree in computer science engineering and telecommunications in May 2004 and Ph.D. in Electronics and Communications Engineering in 2008. He is an assistant professor at DIMES Department of University of Calabria, after many collaborations with the UPV of Valencia and the VSB-Technical University of Ostrava. His research interests include mobile communication networks, QoS architectures and interworking wireless and wired networks, mobility modeling for WLAN environments and mobility analysis for prediction purposes. 